\title{
Adiposidad corporal, estilos de vida y nivel de actividad física en mujeres dueñas de casa y trabajadoras remuneradas chilenas
}

\section{Adiposity, healthy lifestyle and physical activity levels in housewives and women with paid employment in Chile}

\section{RESUMEN}

En Chile existe escasa evidencia en relación a las características de los estilos de vida en mujeres dueñas de casa versus mujeres con trabajos renumerados. El objetivo del estudio fue comparar marcadores de adiposidad, hábitos de consumo asociados a estilos de vida y nivel de actividad física $(A F)$ en mujeres dueñas de casa y trabajadoras remuneradas chilenas. Se compararon variables de adiposidad como el índice de masa corporal (IMC), peso corporal, perímetro de cintura $(P C)$, estilos de vida y niveles de AF (transporte, moderada y vigorosa intensidad y tiempo sedente) de 2.232 participantes de la Encuesta Nacional de Salud 2009-2010. Las dueñas de casa, en comparación con las trabajadoras renumeradas, presentaron un mayor IMC (27,9 vs. 20,0 kg/m², p<0,0001) y PC (98,3 vs. 95,6 $\mathrm{cm}, p<0,0001)$. El tiempo destinado a la práctica de $A F$ de trasporte $(42,5 \mathrm{vs} .51,5 \mathrm{~min} /$ día, $p=0,009)$, moderada (94,2 vs. 128,4 min/día, $p<0,0001)$ y vigorosa (25,06 vs. $53,49 \mathrm{~min} / d i a, p<0,0001)$ fue menor en dueñas de casa que en trabajadoras renumeradas. Por el contrario, las dueñas de casa destinaron menor tiempo al desarrollo de actividades sedentes (2,71 vs. 3,26 horas/día, $p<0,0001)$, además mostraron un menor consumo de alcohol (30,5 vs. 43,2 g/día, $p=0,021$ ) en comparación a trabajadoras renumeradas.

Palabras clave: Actividad física; Adiposidad; Conducta alimentaria; Estilo de vida; Mujer; Obesidad.

\footnotetext{
ABSTRACT

Lifestyle behaviours among women who take care of their home duties (housewives) and women who are currently working in paid employment is unknown in the Chilean population. The aim of this study was, therefore, to characterise adiposity, lifestyle behaviours and physical activity levels in housewives and women with paid employment in Chile. A total of 2,231 women from the National Health Survey 2009-2010 were included in this study. Adiposity markers (body weight, body mass index and waist circumference), lifestyle behaviors and physical activity were measured using self-reported questionnaires. The main findings of this study was that housewives had a higher body mass index (27,9 vs.
}

Yeny Concha-Cisternas ${ }^{1,2}$, Fanny Petermann-Rocha $a^{3,4}$, Jaime A. Vásquez-Gómez ${ }^{5}$, Carlos Matus-Castillo ${ }^{6}$, Ana María Leiva ${ }^{7}$, María Adela Martínez ${ }^{8}$, Alex Garrido-Mendez ${ }^{6,9}$, Ximena Diaz-Martinez ${ }^{10}$, Edgardo Molina-Sotomayor ${ }^{11}$, Gustavo Pavez-Adasme ${ }^{12}$, Hernaldo Carrasco Beltrán ${ }^{13,14}$, Jaime Silva-Urra ${ }^{15}$, Ana Rosa Beltran ${ }^{16}$, Carlos Celis-Morales ${ }^{4,17^{*}}$ en representación del Grupo de Investigación ELHOC.

1. Escuela de Kinesiología, Facultad de Salud, Universidad Santo Tomás, Talca, Chile. 2. Pedagogía en Educación Física, Facultad de Educación Universidad Autónoma de Chile, Chile.

3. BHF Glasgow Cardiovascular Research Centre, Institute of

Cardiovascular and Medical Science, University of Glasgow, Glasgow, United Kingdom.

4. Institute of Health and Wellbeing, University of Glasgow, Glasgow, UK 5. Vicerrectoría de Investigación y Postgrado, Universidad Católica del Maule, Talca, Chile.

6. Departamento de Ciencias del Deporte y Acondicionamiento Físico, Universidad Católica de la Santísima Concepción, Concepción, Chile.

7. Instituto de Anatomía, Histología y Patología, Facultad de Medicina, Universidad Austral de Chile, Valdivia, Chile. 8. Instituto de Farmacia, Facultad de Ciencias, Universidad Austral de Chile, Valdivia, Chile.

9. Departamento de Ciencias del Deporte y Acondicionamiento Físico. Facultad de Educación. Universidad Católica de la Santísima Concepción, Concepción, Chile.

10. Grupo de Investigación en Calidad de Vida, Departamento de Ciencias de la Educación, Facultad de Educación y Humanidades, Universidad del Biobío, Chillan, Chile.

11. Universidad Metropolitana de Ciencias de la Educación, Santiago de Chile. 12. Grupos de Investigación AFSYE, Pedagogía en Educación Física. Universidad Adventista de Chile. Chillán Chile.

13. Facultad de Ciencias de la Actividad Física y del Deporte, Universidad de Playa Ancha, Valparaíso, Chile. 14. Centro de Estudios Avanzados - UPLA. Valparaiso, Chile. 15. Departamento Biomédico, Universidad de Antofagasta, Antofagasta, Chile.

16. Universidad de Antofagasta, Antofagasta, Chile. 17. Centro de Investigación en Fisiología del Ejercicio - CIFE, Universidad Mayor, Santiago, Chile.

* Dirigir correspondencia a: Dr. Carlos Celis-Morales. BHF Glasgow Cardiovascular Research Centre, Institute of Cardiovascular and Medical Sciences, College of Medical, Veterinary and Life Sciences, University of Glasgow, Glasgow G12 8TA, United Kingdom. Tel: 044 (0) 1413304201. Fax: 044 (0) 1413305481 E-mail: carlos.celis@glasgow.ac.uk

Este trabajo fue recibido el 05 de julio de 2018. Aceptado con modificaciones: 01 de abril de 2019. Aceptado para ser publicado: 27 de junio de 2019. 
$\left.20,0 \mathrm{~kg} / \mathrm{m}^{2}, p<0,0001\right)$ and waist circumference $(98,3 \mathrm{vs}$. $95,6 \mathrm{~cm}, p<0,0001)$ than women with paid-employment. Physical activity levels were lower (transport PA 42,5 vs. $51,5 \mathrm{~min} /$ day, $p=0,009$, moderate $P A 94,2$ vs. $128,4 \mathrm{~min} /$ day, $p<0,0001$ and vigorous $P A 25,06$ vs. 53,49 min/day, $p<0,0001)$ and sedentary-related behaviours (2,71 vs. 3,26 hours/day, $p<0,0001)$ were higher in housewives compare to women with paid-employment. Dietary behaviors showed that housewives consume less alcohol (30,5 vs. 43,2 g/day, $p=0,021)$ than women with paid-employment.

Keywords: Adiposity, Eating Behavior; Exercise; Obesity; Lifestyle; Women.

\section{INTRODUCCIÓN}

Durante las últimas tres décadas la salud cardiovascular de la población se ha visto fuertemente deteriorada debido a la adopción de estilos de vida poco saludables y escasa práctica de actividad física (AF), lo que ha conllevando la aparición de enfermedades crónicas no transmisibles (ECNT) $)^{1,2}$.

Resultados de la Encuesta Nacional de Salud (ENS) 2016-2017, evidencian que tanto hombres como mujeres exhiben cambios en sus hábitos y patrones alimentarios, orientados a un mayor consumo de sal, azúcares simples, grasas y menor ingesta de frutas y verduras, además de un elevado consumo de alcohol y tabaco ${ }^{3}$. Estos hábitos se asocian directamente con el desarrollo de enfermedades crónicas, como hipertensión arterial (HTA), diabetes mellitus tipo 2 (DMT2), sobrepeso y obesidad 4 .

Bajo este contexto las mujeres, pero principalmente las dueñas de casa, podrían considerarse una población de alto riesgo para la adquisición y desarrollo de enfermedades cardiovasculares debido a sus hábitos y conductas poco saludables ${ }^{5}$. Investigaciones han señalado que las dueñas de casa autorreportan un estilo de vida sedentario y mantienen una alimentación con bajo consumo de frutas, verduras y agua, además presentan alta prevalencia de sobrepeso, obesidad y un mayor perímetro de cintura (PC) $)^{6,7,8,9,10}$. Por otro lado, estudios internacionales dan cuenta que las dueñas de casa mantienen bajos niveles de AF, principalmente de tipo vigorosa, lo cual se acentúa conforme avanza la edad ${ }^{11,12}$. Este último antecedente no deja de ser preocupante ya que se ha documentado ampliamente los beneficios que confiere la práctica regular de AF sobre la salud de las personas ${ }^{13,14}$.

En contraste, estudios señalan que las mujeres que desarrollan un trabajo remunerado gozan de mejor salud que las dueñas de casa a tiempo completo ${ }^{15}$. Mientras la ejecución de tareas domésticas limita la realización de otro tipo de actividades de ocio y esparcimiento, el entorno laboral proporcionaría mayores oportunidades e independencia económica para acceder a mejores condiciones en términos de alimentación y práctica de $\mathrm{AF}^{15,16}$.

Aunque existe evidencia que da cuenta de los hábitos y conductas de las mujeres dueñas de casa y trabajadoras remuneradas, estas corresponden a investigaciones que se han desarrollado internacionalmente ${ }^{7,8,10}$, por lo que, en Chile, este correspondería al primer estudio en abordar a esta población. En consecuencia, el objetivo de este trabajo fue comparar marcadores de adiposidad, estilos de vida y nivel $\mathrm{AF}$ en mujeres dueñas de casa y trabajadoras remuneradas chilenas.

\section{MATERIALES Y MÉTODOS Diseño del estudio}

La muestra seleccionada incluyó a los participantes de la ENS 2009-2010 de Chile, desarrollada entre octubre del año 2009 y septiembre del año 2010 ${ }^{17}$. Dicha encuesta correspondió a un estudio de prevalencia realizado en hogares a partir de una muestra nacional, probabilística, estratificada y multietápica de 5.412 personas mayores de 15 años con representatividad nacional, regional, y área urbana/rural. En este estudio se incluyó un total de 2.232 participantes mujeres, que tenían información disponible en relación con sus estilos de vida. Fueron excluidos del análisis 26 hombres que reportaron ser dueños de casa sin remuneración. El protocolo de estudio de la ENS 2009-2010 fue aprobado por el Comité de Ética de la Escuela de Medicina de la Pontificia Universidad Católica de Chile. Todos los participantes firmaron un consentimiento informado ${ }^{17}$.

\section{Variables sociodemográficas y hábitos de consumo asociados a de estilo de vida}

Las variables sociodemográficas como edad, sexo, nivel educacional (básica $<8$ años, media 8 a 12 años, educación superior $>12$ años), ingreso económico (Bajo $<250.000$; Medio 250.000 a 450.000; Alto > 450.000 pesos chilenos) y hábitos de consumo asociados a estilos de vida, como el tabaquismo, consumo de alcohol, frutas y verduras, se obtuvieron mediante la aplicación de cuestionarios validados en la ENS 2009-2010 ${ }^{17}$. El consumo de sal se determinó mediante análisis de excreción de sodio en la orina ${ }^{17}$. Para evaluar diferencias entre las variables de estudio se subdividió a las dueñas de casa y trabajadoras remuneradas en 3 grupos de edad: < 40 años; 40-55 años y > 55 años.

\section{Mediciones antropométricas}

El estado nutricional y adiposidad corporal fueron determinados según Índice de Masa Corporal (IMC) en base a los puntos de corte de la Organización Mundial de la Salud (OMS): bajo peso: IMC $<18,5 \mathrm{~kg} / \mathrm{m}^{2}$; normo peso: $18,5-24,9 \mathrm{~kg} / \mathrm{m}^{2}$; sobrepeso: $25,0-29,9 \mathrm{~kg} / \mathrm{m}^{2}$ y obesidad: $\geq 30,0 \mathrm{~kg} / \mathrm{m}^{218}$. La obesidad central para mujeres fue definida como un $\mathrm{PC} \geq 83 \mathrm{~cm}$, según los puntos de corte sugeridos por la ENS 2009-2010 en población chilena ${ }^{17}$.

\section{Clasificación de actividad física}

Los niveles de AF, el tiempo destinado a las actividades de transporte activo (ej. caminar, andar en bicicleta), y las actividades de intensidad moderada o vigorosa de la población, fueron determinados con el cuestionario "Global Physical Activity Questionnaire" Versión 2 (GPAQ v2) ${ }^{19}$, el cual ha sido validado en población latina ${ }^{20}$. La AF total es presentada como la suma del tiempo reportado en actividades 
de transporte, de intensidad moderada y vigorosa tanto en el trabajo como en el tiempo libre. Esta variable fue expresada en equivalentes metabólicos (METS) por minutos/semana. Se consideró como punto de corte para inactividad física un gasto energético menor a 600 Mets/minutos/semana o su equivalente de 150 minutos de $\mathrm{AF}$ de intensidad moderada a vigorosa o 75 minutos de AF de intensidad vigorosa a la semana o su combinación, según las recomendaciones de la OMS y especificaciones de la guía de análisis de GPAQ v219,21 Los niveles de sedentarismo fueron determinados mediante el mismo cuestionario GPAQ v2, a partir del autorreporte de tiempo destinado a actividades que involucren estar sentado o reclinado durante el tiempo libre o de trabajo (ej. tiempo sentado frente al computador o televisión, viajando en bus, tren o auto, entre otras). Se consideró alto nivel de sedentarismo cuando una persona destinaba más de 4 horas a estar sentada al día ${ }^{22}$.

\section{Análisis estadístico}

Las características de la población estudiada para dueñas de casa y trabajadoras renumeradas son presentadas como promedio y su respectiva desviación estándar para variables continuas y como porcentaje para variables de tipo categóricas. También se presentan las características según grupo etario para dueñas de casa y trabajadoras remuneradas. Se establecieron comparaciones formales para estas variables ya que el objetivo de esta tabla es solo caracterizar a la población.

Las diferencias para los niveles de adiposidad, alimentación y AF en dueñas de casa versus mujeres con trabajos renumerados fueron determinados mediante regresión lineal. También se realizaron análisis de tendencia para niveles de adiposidad, alimentación y AF según grupos etarios en dueñas de casa y trabajadoras renumeradas, los que fueron realizados mediante regresión lineal ajustando por factores de confusión. Todos los datos son presentados como promedios y sus respectivos $95 \%$ de intervalos de confianza ( $95 \%$ IC).

Los análisis fueron ajustados por las siguientes variables de confusión: zona de residencia (rural, urbano), nivel educacional y tabaquismo. Las variables de edad, IMC, tiempo sedente, niveles de AF y variables de alimentación (consumo de alcohol, sal, frutas y verduras) fueron solo incluidas en aquellos análisis donde estas variables no correspondían a la variable de salida o donde los análisis no fueron estratificados por edad. Para el procesamiento de todos los datos se utilizó el módulo de muestras complejas del programa STATA SE v14. El nivel de significancia fue definido como $p<0,05$.

\section{RESULTADOS}

Las características socio-demográficas, antropométricas y de estilos de vida se presentan en la tabla 1. Al comparar dueñas de casa con el grupo de trabajadoras remuneradas, las dueñas de casa tienen mayor promedio de edad (47,1 años), un mayor porcentaje habita en zonas rurales, concentran un mayor número de individuos en un nivel educacional bajo-medio, y con un promedio de 8,7 años de escolaridad. Similares características fueron identificadas al estudiar a la población según rango etario (Tabla 2).

En la figura 1 se observan los marcadores de adiposidad (peso corporal, IMC, PC), estilos de vida (consumo de alcohol, sal, frutas y verduras y nivel de AF) en mujeres dueñas de casa y trabajadoras remuneradas. Al comparar a mujeres trabajadoras remuneradas con dueñas de casa, estas últimas exhiben un mayor IMC $\left(28,9 \mathrm{~kg} / \mathrm{m}^{2}\right.$ vs. $\left.28,0 \mathrm{~kg} / \mathrm{m}^{2}\right)$ y PC $(98,3$ $\mathrm{cm}$ vs.95,6 cm). En cuanto al nivel de AF, las dueñas de casa destinan menos tiempo al desarrollo de AF en todos sus tipos (transporte: $42,5 \mathrm{~min} /$ día vs. 51,5 min/día, moderada: 94,2 $\mathrm{min} /$ día vs. $128,4 \mathrm{~min} /$ día y vigorosa: $25,06 \mathrm{~min} /$ día vs. 53,4 $\mathrm{min} /$ día) en comparación con trabajadoras remuneradas. Lo mismo sucede con el tiempo dedicado a realizar actividades sedentes (2,71 hora/día vs. 3,26 hora/día). Finalmente, las mujeres que realizan actividades domésticas muestran un menor consumo de alcohol (30,5 g/día vs. 43,2 g/día), pero no se encontraron diferencias para el consumo de sal, frutas, y verduras.

La figura 2 muestra las variaciones de los marcadores de adiposidad en mujeres dueñas de casa y trabajadoras remuneradas en diferentes rangos etarios. Las dueñas de casa de todos los grupos etarios muestran una tendencia estadísticamente significativa a aumentar el IMC en la medida que se incrementa la edad $(p<0,0001)$. Similar comportamiento ocurre en el grupo de trabajadoras remuneradas $(p=0,003)$. En relación con el PC, en ambos grupos existe un cambio conforme avanza la edad, exhibiendo un aumento estadísticamente significativo en todos los grupos etarios $(p<0,0001)$.

Los hábitos de consumo asociados a estilos de vida en mujeres dueñas de casa y trabajadoras remuneradas, y sus variaciones según grupo etario se observan en la figura 3. El consumo de frutas y verduras en dueñas de casa y trabajadoras remuneradas mostró un aumento significativo a medida que aumenta la edad ( $p=0,028$ y $p=0,003$ respectivamente). Sumado a lo anterior, los resultados revelan que, a mayor edad, se evidencia una tendencia a disminuir el consumo de alcohol, siendo significativa en las trabajadoras remuneradas $(p=0,010)$. En cuanto al consumo de sal, las mujeres dueñas de casa y trabajadoras remuneradas no exhiben cambios significativos en los diferentes grupos etarios.

La figura 4 muestra las variaciones en el tiempo destinado a los diferentes tipos de AF según rango etario en mujeres dueñas de casa y trabajadoras remuneradas. En la medida que aumenta la edad, las dueñas de casa y trabajadoras remuneradas revelan una tendencia a disminuir el tiempo destinado a la práctica de AF de intensidad vigorosa, moderada y de transporte. Finalmente, en todos los rangos etarios, las dueñas de casa no mostraron diferencias significativas en las horas/día destinadas al desarrollo de actividades de tipo sedente $(p=0,897)$, por lo que esta conducta no varía con la edad. Diferente es lo encontrado en el grupo de mujeres trabajadoras remuneradas, en donde los grupos etarios $<44$ años y 44-55 años reportan un mayor tiempo destinado a ésta actividad, sin embargo a partir de los 55 años, existe una tendencia significativa a disminuir el tiempo dedicado a esta conducta $(p=0,002)$. 
Tabla 1. Características socio-demográficas, antropométricas y estilos de vida en dueñas de casa y trabajadoras remuneradas.

\begin{tabular}{|c|c|c|}
\hline Variables & Dueñas de casa & Trabajadoras remuneradas \\
\hline \multicolumn{3}{|l|}{ Sociodemográficas } \\
\hline Total $(\mathrm{n})$ & 1.190 & 1.042 \\
\hline Edad (años) & $47,1 \pm 16,0$ & $41,9 \pm 12,2$ \\
\hline Años de escolaridad & $8,7 \pm 3,9$ & $11,3 \pm 3,9$ \\
\hline \multicolumn{3}{|l|}{ Zona geográfica (\%) } \\
\hline Rural & 24,2 & 7,9 \\
\hline Urbano & 75,8 & 92,1 \\
\hline \multicolumn{3}{|l|}{ Nivel educacional (\%) } \\
\hline Bajo (<8 años) & 33,2 & 13,6 \\
\hline Media (8- 12 años) & 58,4 & 56,5 \\
\hline Alto (> 12 años) & 8,4 & 29,9 \\
\hline \multicolumn{3}{|l|}{ Nivel de ingreso (\%) } \\
\hline Bajo $(<250.000 \mathrm{CLP})$ & 64,3 & 48,8 \\
\hline Medio ( 250.000- 450.000 CLP) & 29,7 & 37,2 \\
\hline Alto $(>450.000 \mathrm{CLP})$ & 6,0 & 14,0 \\
\hline \multicolumn{3}{|l|}{ Antropométricas } \\
\hline Peso corporal $(\mathrm{kg})$ & $69,5 \pm 13,8$ & $69,4 \pm 13,2$ \\
\hline $\mathrm{IMC}\left(\mathrm{kg} / \mathrm{m}^{2}\right)$ & $28,9 \pm 5,5$ & $28,0 \pm 5,6$ \\
\hline \multicolumn{3}{|l|}{ Estado Nutricional IMC (\%) } \\
\hline$<18,5 \mathrm{~kg} / \mathrm{m}^{2}$ & 1,4 & 0,6 \\
\hline $18,5-24,9 \mathrm{~kg} / \mathrm{m}^{2}$ & 23,4 & 30,7 \\
\hline $25,0-29,9 \mathrm{~kg} / \mathrm{m}^{2}$ & 33,8 & 39,0 \\
\hline$\geq 30,0 \mathrm{~kg} / \mathrm{m}^{2}$ & 38,4 & 26,7 \\
\hline Perímetro de Cintura $(\mathrm{cm})$ & $98,1 \pm 12,5$ & $95,6 \pm 12,6$ \\
\hline Obesidad central, n $(\%)$ & 60,1 & 52,5 \\
\hline \multicolumn{3}{|l|}{ Estilos de vida } \\
\hline Actividad física total (MET/hora/semana ) & $87,8 \pm 101,4$ & $133,9 \pm 146,4$ \\
\hline Actividad física de transporte (mín/día) & $43,2 \pm 73,4$ & $51,5 \pm 87,7$ \\
\hline Actividad física moderada (mín/día ) & $93,9 \pm 130,5$ & $128,5 \pm 162,1$ \\
\hline Actividad física vigorosa (mín/día) & $25,5 \pm 71,2$ & $53,5 \pm 122,9$ \\
\hline Tiempo sedente (hora/día ) & $2,7 \pm 1,9$ & $3,3 \pm 2,8$ \\
\hline Físicamente activo (600 Mets/mín/sem) & 73,8 & 80,3 \\
\hline Físicamente inactivo (600 Mets/mín/sem) & 26,1 & 19,7 \\
\hline Horas de sueño (hora/día) & $7,7 \pm 1,7$ & $7,2 \pm 5,0$ \\
\hline Consumo de frutas y verduras (g/día) & $219,6 \pm 138,0$ & $228,0 \pm 140,6$ \\
\hline Consumo de alcohol (g/día) & $31,2 \pm 30,8$ & $43,3 \pm 81,2$ \\
\hline Consumo de sal (g/día) & $9,6 \pm 2,7$ & $9,41 \pm 2,6$ \\
\hline \multicolumn{3}{|l|}{ Tabaquismo (\%) } \\
\hline Nunca & 47,6 & 39,1 \\
\hline Ex-fumador & 21,0 & 20,2 \\
\hline Fumador & 31,4 & 40,7 \\
\hline
\end{tabular}

Datos presentados como media y su respectiva desviación estándar para variables continúas y como porcentaje (\%) para variables categóricas. CLP: pesos chilenos 
Tabla 2. Características de la población por grupo de edad.

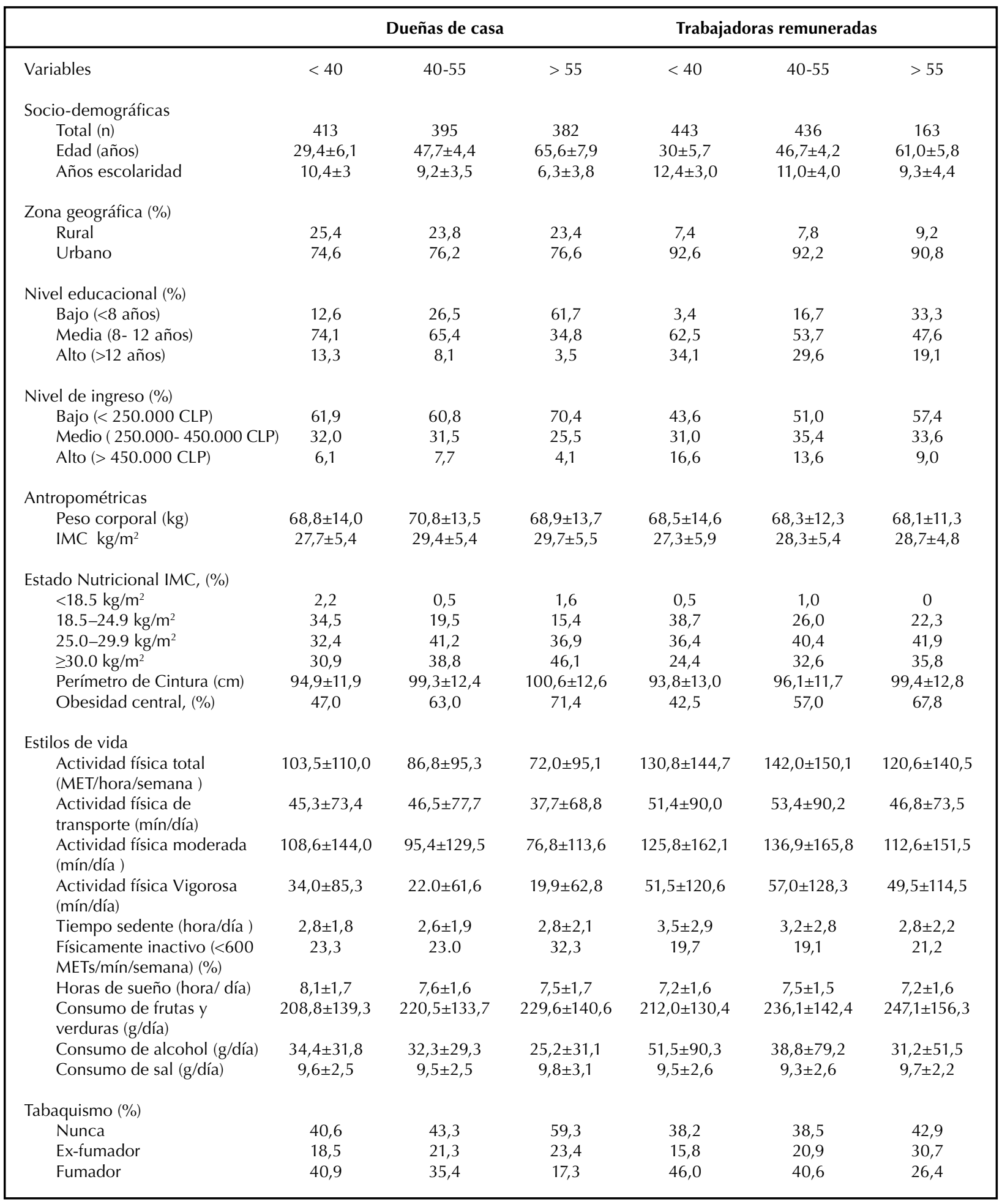

Datos presentados como media y su respectiva desviación estándar para variables continúas y como porcentaje (\%) para variables categóricas. CLP: Pesos chilenos. 

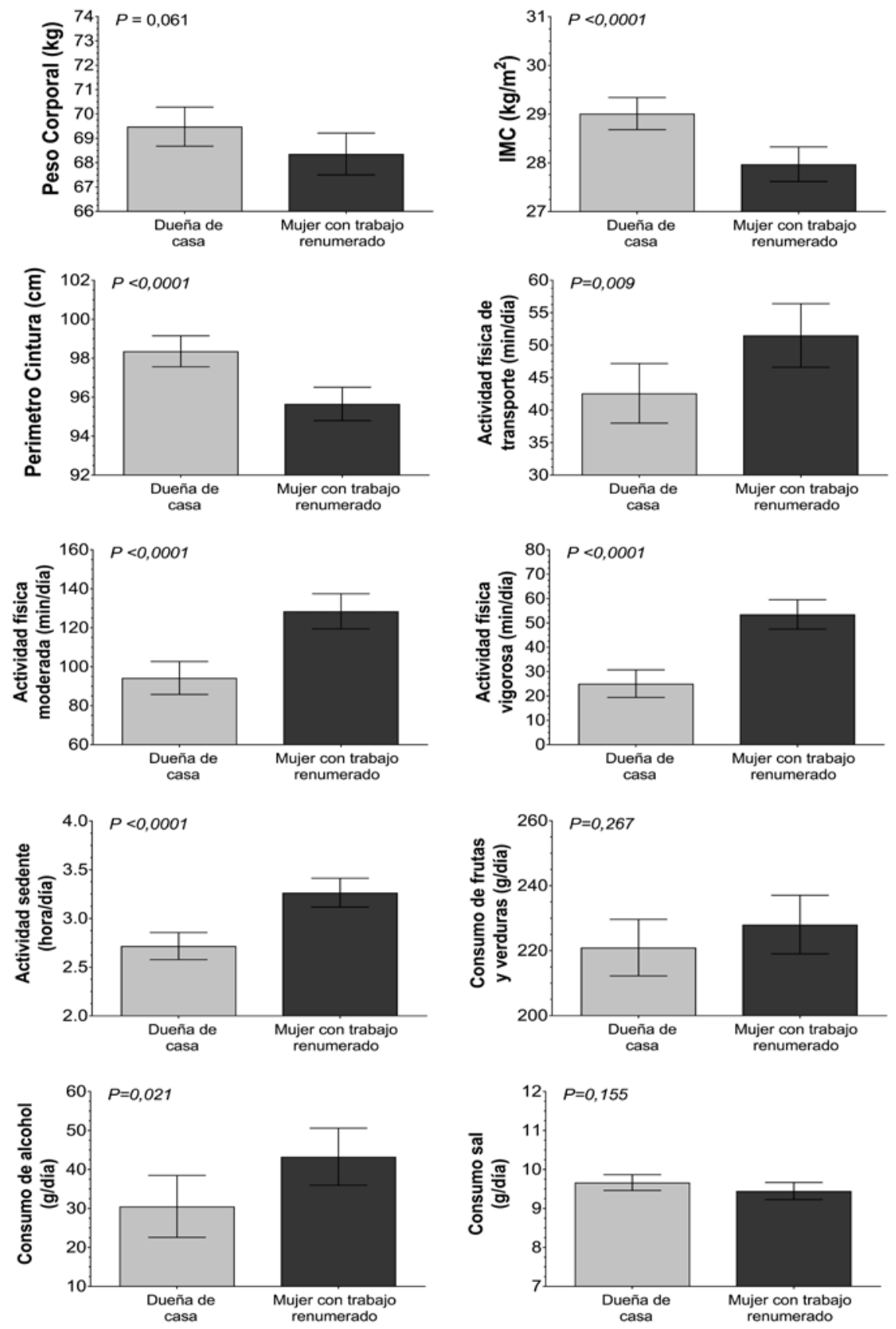

Figura 1: Marcadores de adiposidad, hábitos de consumo asociados a estilos de vida y nivel de actividad física en mujeres dueñas de casa y trabajadoras remuneradas.

Datos presentados como promedio y su respectivo 95\% de intervalos de confianza. Todos los análisis fueron ajustados por edad, zona de residencia, tabaquismo y nivel educacional. Las variables antropométricas fueron ajustadas de forma adicional por tiempo sedente y actividad física. Variables de actividad física fueron ajustadas de forma adicional por IMC y variables de alimentación. Finalmente, las variables de alimentación fueron ajustadas de forma adicional por IMC, tiempo sedente y actividad física. 

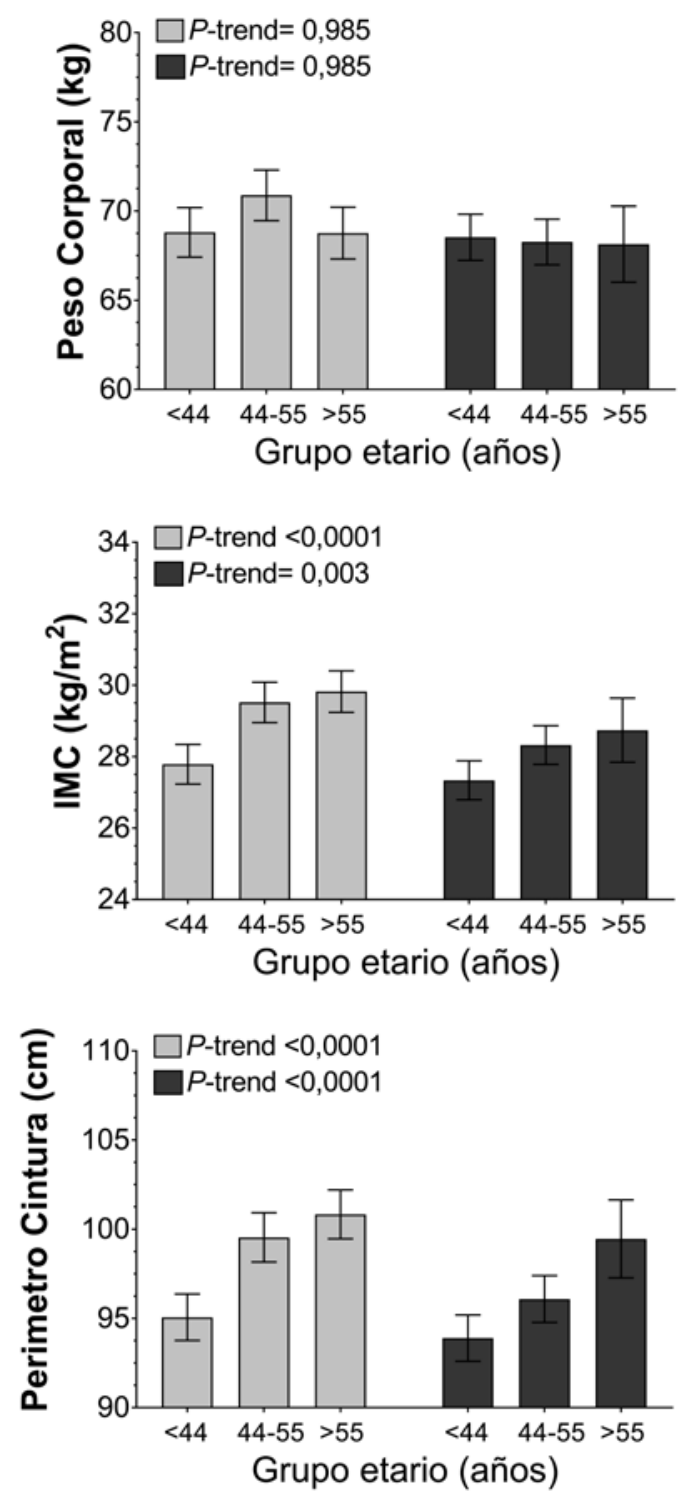

Dueña de casa

Mujer trabajo remunerado

Figura 2: Marcadores de adiposidad en mujeres dueñas de casa y trabajadoras remuneradas en diferentes rangos etarios.

Datos son presentados como promedio y sus respectivos $95 \%$ de intervalos de confianza. "P-trend" indica la variación del peso corporal, IMC y PC en dueñas de casa y trabajadoras remuneradas según rango etario. Todos los análisis fueron ajustados por zona de residencia, nivel educacional, tabaquismo, tiempo sedente y niveles de actividad física.
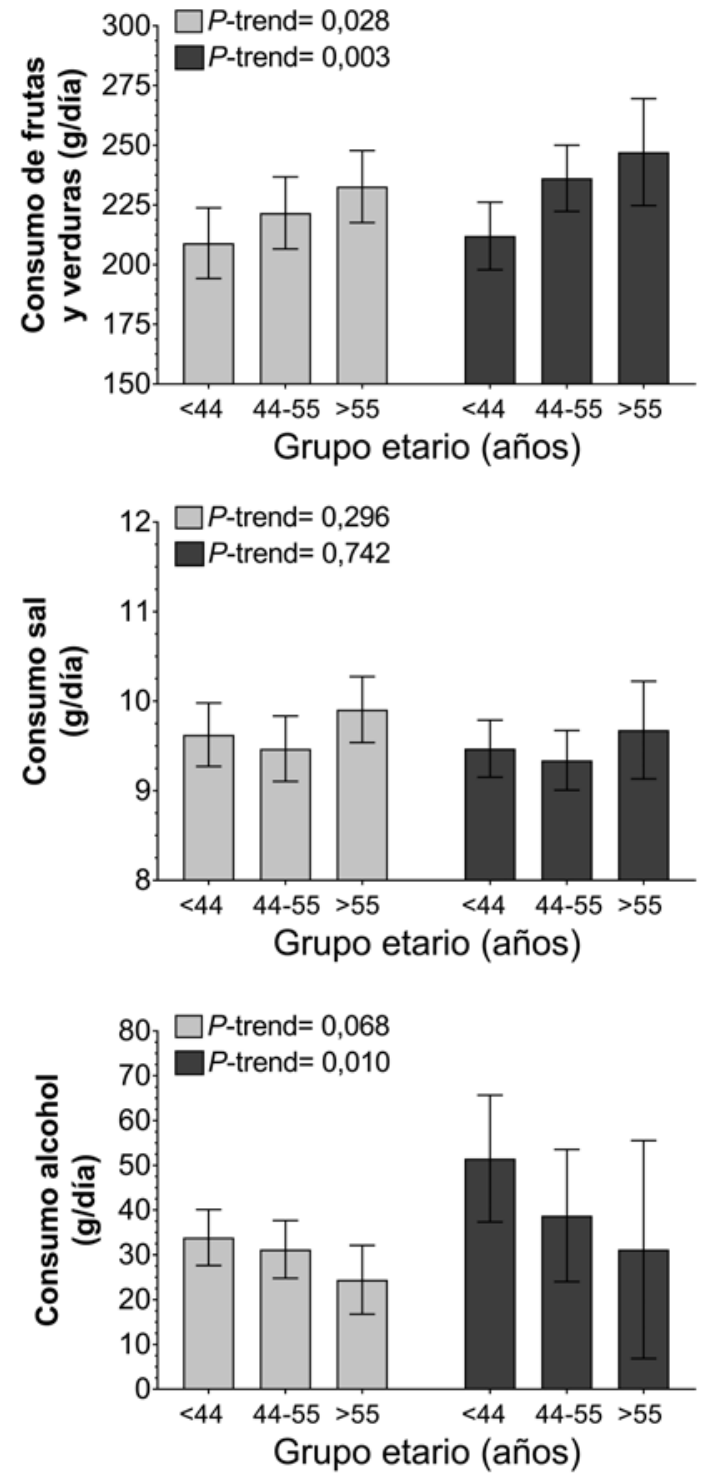

Dueña de casa Mujer trabajo remunerado

Figura 3: Alimentación en mujeres dueñas de casa y trabajadoras remuneradas en diferentes rangos etario. Datos son presentados como promedio y sus respectivos $95 \%$ de intervalos de confianza. "P-trend" indica la variación del consumo de frutas y verduras, sal y alcohol en dueñas de casa y trabajadoras remuneradas según rango etario. Todos los análisis fueron ajustados por zona de residencia, nivel educacional, tabaquismo, IMC, tiempo sedente $y$ niveles de actividad física. 

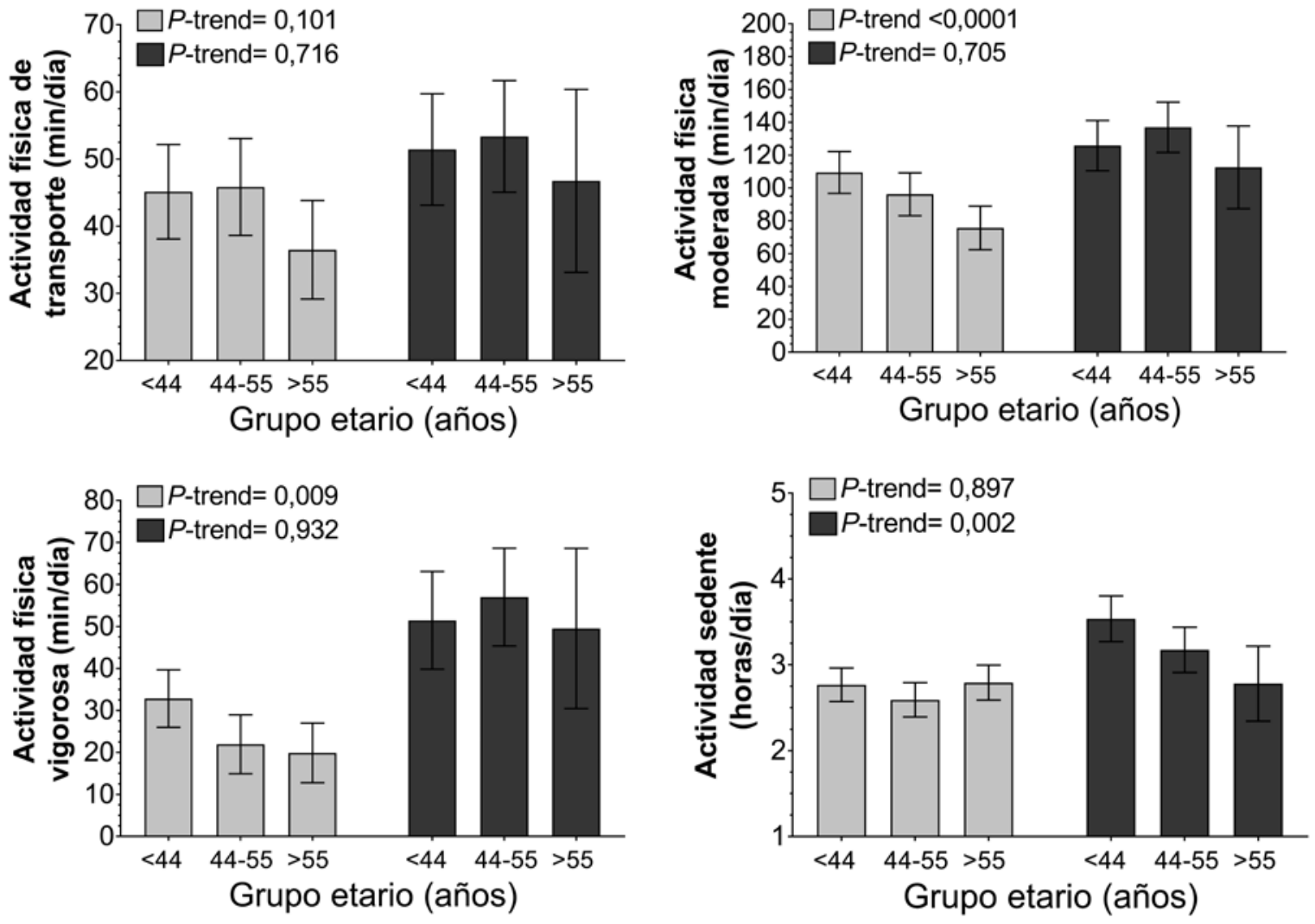

Dueña de casa

Mujer trabajo remunerado

Figura 4: Tiempo destinado para cada intensidad de actividad física según rango etario en mujeres dueñas de casa y trabajadoras remuneradas.

Datos son presentados como promedio y sus respectivos 95\% de intervalos de confianza. "P-trend" indica la variación los niveles de AF en dueñas de casa y trabajadoras remuneradas según rango etario. Todos los análisis fueron ajustados por zona de residencia, nivel educacional, tabaquismo, IMC y alimentación.

\section{DISCUSIÓN}

Los principales resultados de este estudio revelan que las mujeres dueñas de casa tienen un mayor nivel de adiposidad y presentan hábitos de consumo poco saludables, además de niveles de AF más bajos en comparación con trabajadoras remuneradas.

Recientemente Raza y Cols., en un estudio que incluyó 600 mujeres, reportaron que las dueñas de casa presentan peor estado nutricional cuantificado a través del IMC, en comparación con sus pares remuneradas?. De igual manera, Ersoy y Imamoglu demostraron que la prevalencia de sobrepeso y obesidad era más alta en mujeres dueñas de casa que en mujeres trabajadoras remuneradas $(42,2 \%$ y $16,6 \%$ respectivamente ${ }^{23}$. En cuanto al PC, la literatura ha revelado valores más altos en mujeres que desarrollan actividades domésticas ${ }^{6,23}$, resultados similares a los encontrados en nuestro estudio.

Actualmente el IMC y PC han demostrado gran validez como marcadores de adiposidad y riesgo metabólico, además se han asociado directamente con el desarrollo 
de comorbilidades, enfermedades cardiovasculares y mortalidad $^{24,25}$. Frente a esta situación es necesario que entidades de salud, deporte y educación puedan intervenir en la creación y/o fortalecimiento de estrategias y programas públicos, orientados a la reducción del peso corporal, IMC y PC de la población considerada vulnerable, por ejemplo las dueñas de casa.

En relación con los hábitos de consumo asociados a estilos de vida, las dueñas de casa reportaron un menor consumo de frutas y verduras, además de un mayor consumo de sal. Datos similares se han identificado en la literatura, tanto en población oriental ${ }^{7,10}$ como occidental $^{6}$. Estilos de vida poco saludables como mantener un bajo consumo de frutas y verduras, y una elevada ingesta de sal se asocian al desarrollo de enfermedades cardiovasculares como DMT2, HTA, y obesidad ${ }^{4,26}$. Este antecedente coincide con las características de la población estudiada, en donde precisamente, las dueñas de casa fueron quienes reportaron mayor prevalencia de estas enfermedades.

Nuestros resultados también indican que las mujeres que desarrollan trabajo remunerado mantienen un mayor consumo de alcohol. Esto podría explicarse por la globalización, el fácil acceso al alcohol por gozar de remuneraciones, a los roles adquiridos por las mujeres en el entorno laboral y social, altos niveles de estrés, entre otros ${ }^{27,28}$.

Por otro lado, el tiempo destinado a la práctica de AF de transporte, moderada y vigorosa, este fue menor en el grupo de mujeres dueñas de casa; no obstante, en ambos grupos, la AF disminuyó en la medida que avanzó la edad. Este hallazgo podría explicarse debido a los cambios relacionados al envejecimiento, los cuales reducirían la ejecución de ciertas actividades motrices ${ }^{29,30}$. De igual manera, las altas demandas laborales y domésticas podrían restringir el tiempo libre dedicado al desarrollo de actividades de ocio, como la práctica de AF.

La OMS establece que las personas entre 18 y 64 años tienen la oportunidad de mantenerse físicamente activas en cuatro sectores principales de la vida diaria: el trabajo, el transporte, tiempo libre o de ocio y las tareas domésticas ${ }^{21}$. De acuerdo a esto, las dueñas de casa se beneficiarían de las actividades domésticas que realizan diariamente, independiente de sus niveles de AF. Asimismo, estudios han señalado que las mujeres que realizan $\mathrm{AF}$ a través de la ejecución de tareas domésticas, disminuyen el riesgo de mortalidad por todas las causas ${ }^{31,32}$. No obstante, estos beneficios no han sido reportados en personas que desempeñan un trabajo remunerado ${ }^{33}$.

Si bien las pautas actuales de AF enfatizan en la práctica regular de al menos 150 minutos de AF moderada o 75 minutos de AF vigorosa semanalmente para mantener o mejorar la salud ${ }^{34}$, también se recomienda que las personas que no logren cumplir con dichas recomendaciones puedan mantenerse físicamente activos en otros dominios o sectores de la vida diaria.

En relación con el autorreporte de tiempo destinado a actividades que involucren estar sentado durante el tiempo libre o de trabajo ( $\geq 4$ horas/día), éste fue mayor en las mujeres que desarrollan trabajo remunerado en todos los rangos etarios, lo que podría relacionarse con el tipo de trabajo y labores que realizan.

Si bien las trabajadoras remuneradas presentan niveles de AF superior a lo reportado por dueñas de casa, también dedican mayor tiempo del día al desarrollo de actividades en sedentes, comportamiento considerado como un factor de riesgo por su asociación con el desarrollo de DMT2 y deterioro de la salud, independiente de los niveles de $\mathrm{AF}^{30,35,36}$. En contraste, la ejecución de tareas domésticas evitaría el desarrollo de conducta sedentaria, pudiendo considerarse un factor protector para la salud de este grupo en particular.

Finalmente, las dueñas de casa reportaron un menor nivel educativo que las mujeres trabajadoras remuneradas. En relación a ello, la literatura ha informado que el nivel educacional está inversamente relacionado con hábitos alimenticios y actividades físicas poco saludables ${ }^{23}$, lo que permitiría explicar en parte, los elevados valores en los marcadores de adiposidad en las dueñas de casa.

Estudios internacionales han sugerido que las mujeres dueñas de casa deben ser consideradas dentro de las políticas gubernamentales por tratarse de una población vulnerable y de alto riesgo, principalmente por la adopción de estilos de vida poco saludable y conducta sedentaria ${ }^{15}$. En Chile, este es el primer estudio que muestra las diferencias entre marcadores de adiposidad, hábitos de consumo asociados a estilos de vida y nivel de AF entre mujeres dueñas de casa y trabajadoras remuneradas, generando evidencia que podría servir de base para la creación y/o fortalecimiento de políticas y programas saludables orientados a dicha población.

Entre las fortalezas de este estudio está la representatividad a nivel nacional de las participantes con un rango amplio de edad, además de tratarse el primer estudio nacional que incluye dueñas de casa y trabajadoras remuneradas. Dentro de las limitaciones se encuentra la recolección de datos mediante cuestionarios, los cuales a pesar de ser validados, podrían no presentar las condiciones reales de las participantes, siendo susceptibles a sesgos relacionados a una sobreestimación o subestimación de las conductas encuestadas $^{37}$. Por otra parte, es importante considerar que debido al diseño observacional de corte transversal de la ENS 2009-2010, no se puede establecer una relación de causa-efecto.

En conclusión, las mujeres dueñas de casa exhiben elevados marcadores de adiposidad, una mayor prevalencia de estilos de vida poco saludables y menores niveles de AF en comparación con trabajadoras remuneradas.

\section{BIBLIOGRAFÍA}

1. Díaz X, Garrido A, Martínez MA, Leiva AM, Álvarez C, Ramírez-Campillo $R$, et al. Correlates of physical inactivity: Findings from the Chilean National Health Survey 2009-2010. Rev Med Chil 2017; 145(10): 1259-1267. 
2. Celis-Morales C, Salas C, Alduhishy A, Sanzana R, Martinez MA, Leiva A, et al. Socio-demographic patterns of physical activity and sedentary behaviour in Chile: results from the National Health Survey 2009-2010. J Public Health 2016; 38(2): E98-E105.

3. MINSAL. National Health Survey 2016-2017: first results. Ministerio de Salud, Gobierno de Chile. Disponible en: http://www.minsal.cl/wp-content/uploads/2017/11/ENS2016-17_PRIMEROS-RESULTADOS.pdf

4. Petermann F, Durán E, Labraña AM, Martínez MA, Leiva AM, Garrido-Méndez A, et al. Risk factors for obesity: analysis of the 2009-2010 Chilean health survey. Rev Med Chil 2017; 145(6): 716-722.

5. O'Donnell CJ, Elosua R. Cardiovascular Risk Factors. Insights from Framingham Heart Study. Rev Esp Cardiol 2008; 61(3): 299-310.

6. Jacoby $E$, Goldstein J, López A, Núñez E, López T. Social class, family, and life-style factors associated with overweight and obesity among adults in Peruvian cities. Prev med 2003; 37(5): 396-405.

7. Raza L, Ali TM, Hasnain A. Comparison of dietary practices and body mass index among educated housewives and working women in karachi. JAMC 2017; 29(2): 293-297.

8. Park K, Choi Y. A study on prevalence of obesity and its related factors in housewives residing in apartments in Taegu. Korean I Nutr 1990; 23(3): 170-178.

9. Ainy E, Azizi F. Women, occupation and cardiovascular risk factors: findings from the Tehran Lipid and Glucose Study. Public health 2007; 121(12): 950-953.

10. Navadeh S, Sajadi L, Mirzazadeh A, Asgari F, Haghazali M. Housewives' obesity determinant factors in iran; national survey-stepwise approach to surveillance. Iran J Public Health 2011; 40(2): 87.

11. Concha-Cisternas Y, Peterman-Rocha F, Garrido-Méndez A, Díaz-Martínez A, Leiva AM, Salas-Bravo C, Martínez-Sanguinetti MA, Iturra-González JA, Matus C, Vásquez Gómez J, CelisMorales C. Patterns of physical activity in Chilean adults across the lifespan. Nutr Hosp 2019; 36(1): 149-158.

12. Hamrik Z, Sigmundová D, Kalman M, Pavelka J, Sigmund E. Physical activity and sedentary behaviour in Czech adults: results from the GPAQ study. Eur J Sport Sci 2014; 14(2): 193-198.

13. Celis-Morales C, Salas C, Álvarez C, Aguilar Farías N, Ramírez Campillos R, Leppe J, et al. Higher physical activity levels are associated with lower prevalence of cardiovascular risk factors in Chile. Rev Med Chil 2015; 143: 1435-1443.

14. Nocon M, Hiemann $T$, Müller-Riemenschneider $F$, Thalau $F$, Roll S, Willich SN. Association of physical activity with all-cause and cardiovascular mortality: a systematic review and meta-analysis. Eur J Cardiovasc Prev Rehabil 2008; 15(3): 239-246.

15. Artazcoz La, Borrell C, Benach J, Cortes I, Rohlfs I. Women, family demands and health: the importance of employment status and socio-economic position. Soc Sci Med 2004; 59(2): 263-274.

16. Annandale E, Hunt K. Gender inequalities in health: research at the crossroads. 2000.

17. MINSAL. National Health Survey 2009-2010. Ministerio de Salud, Gobierno de Chile. 2010. Disponible en: http://www.minsal. cl/portal/url/item/bcb03d7bc28b64dfe040010165012d23. $p d f$

18. WHO. Obesity: Preventing and managing the Global Epidemic. 2000. http://www.who.int/nutrition/publications/obesity/
WHO TRS 894/en/

19. WHO. Global Physical Activity Questionnaire: GPAQ version 2.0. World Health Organization. 2009. Disponible en: http:// www.who.int/chp/steps/resources/GPAQ_Analysis_Guide. pdf.

20. Hoos T, Espinoza N, Marshall S, Arredondo EM. Validity of the Global Physical Activity Questionnaire (GPAQ) in Adult Latinas. J Phys Act Health 2012; 9(5): 698-705.

21. WHO. Global recommendations on physical activity for health. World Health Organization. 2010. Available: http://www. who.int/dietphysicalactivity/factsheet_recommendations/ en/.

22. Celis-Morales C, Salas C, Alduhishy A, Sanzana R, Martinez $M A$, Leiva $A$, et al. Socio-demographic patterns of physical activity and sedentary behaviour in Chile: results from the National Health Survey 2009-2010. J. Public Health 2016; 38(2): e98-e105.

23. Ersoy C, Imamoglu S. Comparison of the obesity risk and related factors in employed and unemployed (housewife) premenopausal urban women. Diabetes Res Clin Pract. 2006; 72(2): 190-196.

24. Lim SS, Vos T, Flaxman AD, Danaei G, Shibuya K, AdairRohani $H$, et al. A comparative risk assessment of burden of disease and injury attributable to 67 risk factors and risk factor clusters in 21 regions, 1990-2010: a systematic analysis for the Global Burden of Disease Study 2010. The lancet. 2012; 380(9859): 2224-2260.

25. Britton KA, Massaro JM, Murabito JM, Kreger BE, Hoffmann $U$, Fox CS. Body fat distribution, incident cardiovascular disease, cancer, and all-cause mortality. I Am Coll Cardiol 2013; 62(10): 921-925.

26. Weschenfelder Magrini D, Gue Martini J. Hypertension: main modifiable risk factors in the family health strategy. Enfermería Global 2012; 11(26): 344-353.

27. Alonso-Castillo MM, Álvarez-Bermúdez J, López-García KS, Rodríguez-Aguilar L, Alonso-Castillo M, Angélica-Armendáriz N. Personal, Psychosocial Risk Factors, and Alcohol Consumption in Adult Women. Investigación en Enfermería: Imagen y desarrollo 2009; 11(1): 97-114.

28. Góngora Soberanes J, Leyva Piña MA. Alcoholism from a gender perspective. El Cotidiano. 2005(132).

29. De Jaeger C. Physiology of aging. EMC-Kinesiterapia-Medicina Física 2011; 32(3): 1-8.

30. Hallal PC, Andersen LB, Bull FC, Guthold R, Haskell W, Ekelund $U$, et al. Global physical activity levels: surveillance progress, pitfalls, and prospects. The lancet 2012; 380(9838): 247-257.

31. Autenrieth CS, Baumert I, Baumeister SE, Fischer B, Peters A, Döring A, et al. Association between domains of physical activity and all-cause, cardiovascular and cancer mortality. Eur J Epidemiol 2011; 26(2): 91-99.

32. Chen L-J, Fox KR, Ku P-W, Sun W-J, Chou P. Prospective associations between household-, work-, and leisure-based physical activity and all-cause mortality among older Taiwanese adults. Asia Pac J Public Health 2012; 24(5): 795-805.

33. Wanner M, Tarnutzer S, Martin BW, Braun J, Rohrmann S, Bopp $M$, et al. Impact of different domains of physical activity on cause-specific mortality: a longitudinal study. Prev Med 2014; 62: 89-95.

34. Huerta JM, Chirlaque MD, Tormo MJ, Buckland G, Ardanaz E, Arriola L, et al. Work, household, and leisure-time physical activity and risk of mortality in the EPIC-Spain cohort. Prev Med 2016; 85: 106-112. 
35. Joseph JJ, Echouffo-Tcheugui JB, Golden SH, Chen H, Jenny NS, Carnethon MR, et al. Physical activity, sedentary behaviors and the incidence of type 2 diabetes mellitus: the Multi-Ethnic Study of Atherosclerosis (MESA). BMJ Open Diabetes Res Care. 2016;4(1): e000185.

36. Owen N, Healy GN, Matthews CE, Dunstan DW. Too much sitting: the population-health science of sedentary behavior. Exerc Sport Sci Rev 2010; 38(3): 105.

37. Marsaux CF, Celis-Morales C, Hoonhout J, Claassen A, Goris A, Forster H, et al. Objectively measured physical activity in European adults: cross-sectional findings from the Food4Me Study. PloS one. 2016; 11(3): e0150902. 